\title{
Influence of polarization azimuth on two-photon absorption in $\mathrm{CdS}$
}

\author{
N.R. Kulish, M.P. Lisitsa, N.I. Malysh \\ V. Lashkaryov Institute of Semiconductor Physics, NAS of Ukraine, 45, prospect Nauky, 03028 Kyiv, Ukraine
}

\begin{abstract}
To study the dependence of the two-photon absorption coefficient $\beta$ on a polarization azimuth $\varphi$ we used the method of one light source at the ruby laser frequency. It was shown that $\beta$ changes smoothly with increasing $\varphi$ from zero to $\pi / 2$. The minimum value of $\beta$ was realized in the vicinity of $\varphi=45^{\circ}$.
\end{abstract}

Keywords: polarization, two-photon absorption, CdS, azimuth of polarization.

Manuscript received 01.08.05; accepted for publication 25.10.05.

\section{Introduction}

A two-photon absorption is the principal reason of losses of the light intensity when light spreads in the elements of power optics [1, 2]. The two-photon absorption coefficient $\beta$ is the quantitative measure of these losses. In most experimental works, in which the two-photon absorption in one-axis crystals was measured, the value $\beta$ was determined either for a direction of the light wave electric vector $\mathbf{E}$ that was parallel $(\mathbf{E} \| \mathbf{C})$ or perpendicular $(\mathbf{E} \perp \mathbf{C})$ to the crystal optical axis $(\mathbf{C})$ [35)]. It is determined that the values of $\beta$ are different for $\mathbf{E} \| \mathbf{C}$ and $\mathbf{E} \perp \mathbf{C}$. The theory of influence of the light polarization on the value of two-photon absorption in the hexagonal $\mathrm{A}^{2} \mathrm{~B}^{6}$ crystals and in the $\mathrm{A}^{3} \mathrm{~B}^{5}$ semiconductors have been given in $[6,7]$. The experimental data about the influence of the polarization azimuth $\varphi$ on the $\beta$ value are not complete. In this work, the dependence $\varphi$ versus $\beta$ was investigated in detail using a typical oneaxial crystal of $\mathrm{CdS}$ as an example. The CdS crystal often serves as a model object in researches of the nonlinear optical phenomena in crystals.

\section{Method and results}

In our experiments, we used 20-ns pulses at $694.3 \mathrm{~nm}$ wavelength from a ruby laser. The Glann prism was used to set linear light polarization. The polarization azimuth of $\varphi$ was determined using the rotating Glann prism. The optical axis of CdS single crystal was placed in a plane surface. The thickness of CdS sample was equaled to $5 \mathrm{~mm}$. The method of one light source was used to determine $\beta$. The ЭЛУ-ФТ photomultipliers was used to measure the input $\left(I_{0}\right)$ and output $(I)$ intensities. The value $I_{0}$ was changed by the set of the calibrated neutral grey filters.

Influence of systematic errors on the value $\beta$ was reduced to minimum in the following way $[5,8]$ : the light beam had nearly cylindrical form, the distribution of light intensity on the cross-section area was nearly homogeneous; the reflection of light from the back side of crystal was taken into account; the transmission value was averaged by $10-15$ pulses with the same distributing of pulse intensity in time.

For all values of polarization azimuth experimental dependence of reverse transmission $1 / T=I_{0} / I$ on $I_{0}$ (points) was similar to that shown in Fig. 1. For its approximation, the following formula was used [3-5]:

$\frac{1}{T}=\frac{I_{0}}{I}=\frac{\exp (K d)}{(1-R)^{2}}+\frac{\beta[\exp (K d)-1]}{K(1-R)}$,

where $K$ and $\beta$ are the coefficients of single and twophoton absorption, $d$ is the thickness, $R$ is the reflection coefficient. Formula (1) (Fig. 1, solid line) describes well the experimental dependence $1 / T=f\left(I_{0}\right)$ (Fig. 1,

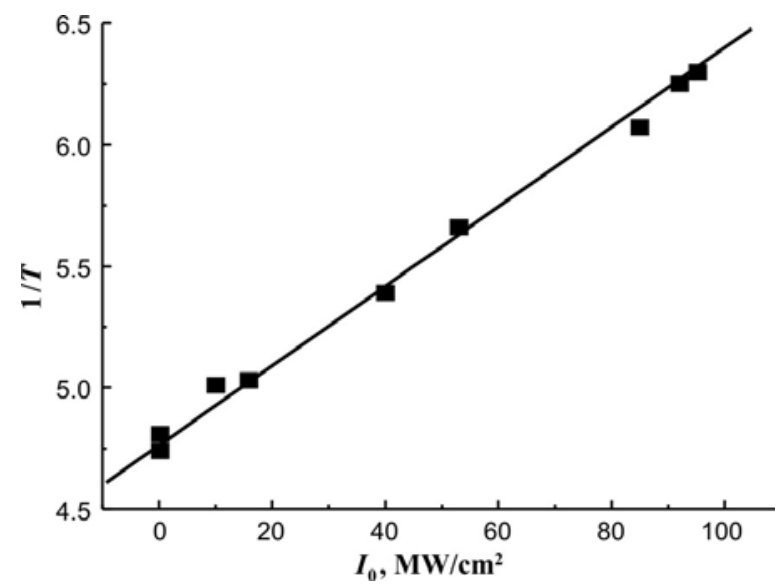

Fig. 1. Variation of the reverse nonlinear transmission $1 / T$ with the intensity $I_{0}$ of linearly polarized ruby laser radiation. The thickness of CdS samples equals $5 \mathrm{~mm}$. The polarization azimuth $\varphi=15^{\circ}$. Points are the experiment, solid line - calculation by the formula (1). 


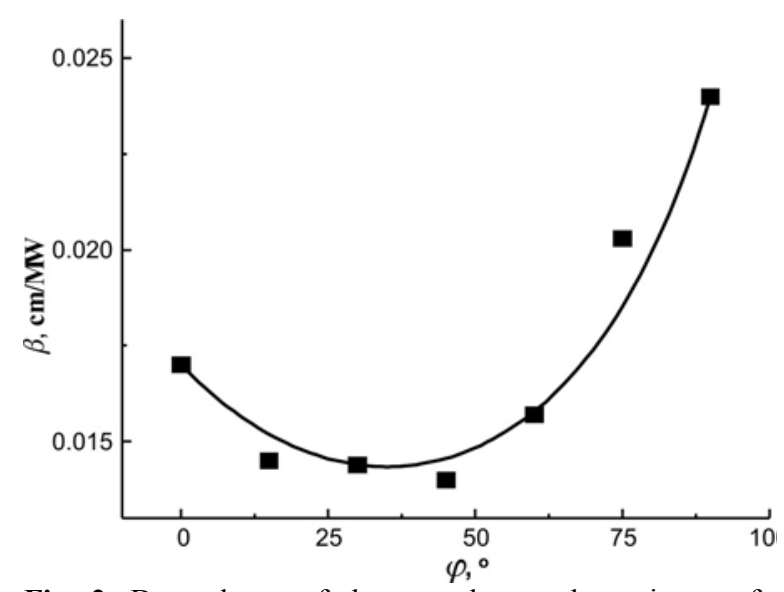

Fig. 2. Dependence of the two-photon absorption coefficient $\beta$ on the azimuth of polarization $\varphi$. Points are the experiment, solid curve is the calculation.

points). The point intersection of solid line (Fig. 1) with $y$-axis determined a constant A. From this constant, we estimated the single-photon absorption coefficient $K=$ $(1 / d) \ln \left[\mathrm{A}(1-R)^{2}\right]$. The slopping angle of solid line determined a constant B. From this constant, we estimated the two-photon absorption coefficient $\beta=$ $[\mathrm{B} K(1-R)] /\left[\mathrm{A}(1-R)^{2}-1\right]$.

It was found that, within the limits of ten percent error, the coefficient of single-photon absorption does not depend on the polarization azimuth. The coefficient of two-photon absorption has the clear pronounced dependence on the polarization azimuth (Fig. 2). There is a qualitative agreement with the theoretical curve [6]. This dependence has been explained in such a way. If the azimuth of the polarization $\varphi \neq n \pi / 2$, where $n=0,1$, $2,3, \ldots$, it is possible to consider that, in one-axis crystal, two light beams travel in the same direction. At the input into a crystal, the intensity of the component with $\mathbf{E} \perp \mathrm{C}$ is $I_{Y O}=I_{0} \sin ^{2} \varphi$, but the component with $\mathbf{E} \| \mathrm{C}$ is $I_{X O}=$ $I_{0} \cos ^{2} \varphi$. These components set a parametric

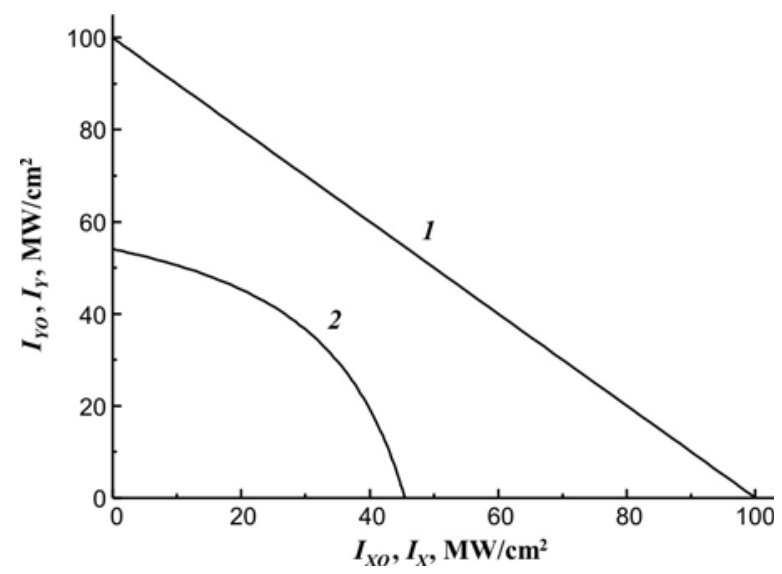

Fig. 3. Parametric dependence of input (1) and output (2) intensities on the polarization azimuth $\varphi$. representation of the dependence $I_{0}$ on $\varphi$ (Fig. 3, curve 1). In accordance with (1), when these beams travel in a crystal, their intensities decreases and output intensities become equal to $I_{Y}$ and $I_{X}$, accordingly. The $I_{Y}$ and $I_{X}$ components set a parametric representation of the dependence $I$ on $\varphi$ (Fig. 3, curve 2). It is clear that the most pronounced change of $I$ takes place at $\varphi=n \pi / 2$ and the least one - near $\varphi=n \pi / 4$. In accordance with (1), the largest changes of the intensity are due to the largest changes of $I_{0} / I$ and $\beta$.

\section{Conclusions}

We have demonstrated the dependence of two-photon absorption coefficient on the polarization azimuth of pumping radiation. The presence of this dependence has to be taken into account at planning and using the power optics elements, which are made of one-axis crystals.

\section{References}

1. P. Liu, W.L. Smith, H. Lotem, J.H. Bechtel, N Blombergen, R.S. Adhav, Absolute two-photon absorption coefficients at 355 and $266 \mathrm{~nm} / /$ Phys. Rev. B 17(12), p. 4620-4632 (1978).

2. V.G. Dmitriev, V.A. Konovalov, Influence of twophoton absorption of radiation on the generation of the second harmonics in crystals // Kvantovaya electronica, 8(3), p. 500-505 (1979)(in Russian).

3. M.S. Brodin, Д.B. Goer, Z.A. Demidenko et al., Two-photon absorption of the mixed semiconductors crystals of $\mathrm{A}^{2} \mathrm{~B}^{6} / /$ Kvantovaya electronica, N 10, p. 56, Naukova dumka, Kiev (1976) (in Russian).

4. V.A Korneichuk, M.P. Lisitsa, I.V. Fekeshgazi, Influence of laser radiation polarization on twophoton absorption of light in the semiconductors of $\mathrm{A}^{2} \mathrm{~B}^{6} / /$ Fizika i tekhnika poluprovodnikov, 11(1), p. 192-195 (1977) (in Russian).

5. M.E. de Souza, Cid B. de Araujo. Two-photon absorption in hexagonal CdS // Solid State Communs 48(11), p. 967-970 (1983).

6. E.B. Beregulin, D.P. Dvornikov, E.L. Ivchenko, I.D. Iaroshetskii, Polarizations properties and linearly-circular dichroism at nonlinear absorption of light in the semiconductors of $\mathrm{A}^{2} \mathrm{~B}^{6} / /$ Fizika $i$ tekhnika poluprovodnikov 9(5), p. 876-885 (1975) (in Russian).

7. E.B. Beregulin, D.P. Dvornikov, E.L. Ivchenko, I.D. Iaroshetskii, Linearly-circular dichroism in the crystals of $\mathrm{A}^{3} \mathrm{~B}^{5}$ near-by the edge of two-photon absorption // Fizika i tekhnika poluprovodnikov 12(8), p. 1571-1576 (1978) (in Russian).

8. R.L. Swofford, W.M. NcClan. The effect of spatial and temporal laser beam characteristics on two-photon absorption // Chem. Phys. Lett. 34(3), p. 455-460 (1975). 